\title{
Ademetionine in Different Combination Regimens to Treat Rosacea: Results from a Randomized, Prospective Clinical Trial
}

\author{
Sviatenko T.V ${ }^{1^{*}}$, Kharitonova O.G ${ }^{2}$, Starostina O.A \\ ${ }^{1}$ Dniepropetrovsk Medical Academy, Dniepropetrovsk, Ukraine \\ ${ }^{2}$ Dnipropetrovsk State Cosmetology Hospital, Dniepropetrovsk, Ukraine \\ ${ }^{3}$ Medical Center PP "Lenomed”, Dniepropetrovsk, Ukraine
}

"Corresponding author: Tetiana Sviatenko, Dnepropetrovsk Medical Academy, 1a 49000 Dniepropetrovsk, Ukraine, Tel: 056 735-20-35; E-mail: tsvyat@rambler.ru

\begin{abstract}
Introduction: Rosacea is characterized by transient or persistent facial erythema, telangiectasia, papules and pustules and is classified into 4 subtypes: erythematoteleangiectatic (stage I), papulopustular (stage II), phymatous (stage III), and ocular rosacea.

Objective: The study assessed the efficacy of different combinations of oral and topical medications, combined or not with ademetionine, in rosacea.

Methods: This randomized, open label, prospective and exploratory study was conducted in 110 healthy male subjects of at least 18 years of age with stage I, stage II or stage III rosacea. Efficacy and safety of different basic (BCR) and complex (basic regimen + ademetionine, CCR) combinations of oral and topical rosacea medications were assessed throughout histopathological, immunohistochemical and clinical methods.

Results: Multiple combination regimens using topical and oral medications significantly improved stage I and stage II/III rosacea. Improvement on a histological, immunohistochemical and clinical level was significantly superior $(p<0.05)$ when ademetionine was added.

Recurrence of stage I or stage II/III rosacea was significantly less frequent $(\mathrm{p}<0.05)$ in patients who received the CCR treatment regimens.

No systemic adverse event was observed. Irritation was reported in 9 patients in the stage I group during the first 3 to 7 days of application of azelaic acid 15\% gel: 5 patients $(16.7 \%)$ in the CCR group, and 4 patients (20\%) in the BCR group. No local adverse events were reported in the stage II/III patient group.

Conclusion: Ademetionine may be a sparring partner in the treatment of rosacea using combination treatments.
\end{abstract}

Received date: January 21, 2016

Accepted date: February 9, 2016

Published date: February 13, 2016

Citation: Sviatenko T.V., et al. Ademetionine in Different Combination Regimens to Treat Rosacea: Results from a Randomized, Prospective Clinical Trial. (2016) Invest Dermatol Venereol Res 2(1): 52- 57.

DOI: $10.15436 / 2381-0858.16 .758$

Keywords: Ademetionine; Rosacea; Combination treatment; Sensibio

\section{Introduction}

Rosacea is one of the most common chronic inflammatory dermatological diseases occurring more frequently in fairskinned individuals, impacting the patients' quality of life ${ }^{[1,2]}$. It is characterized by transient or persistent facial erythema, telangiectasia, papules and pustules, usually on the central portion of the face, and can be classified into 4 main subtypes: erythemato-teleangiectatic (stage I), papulopustular (stage II), phymatous (stage III), and ocular rosacea ${ }^{[3-5]}$.

The exact cause of its pathogenesis is not yet well understood and several hypotheses have been raised ${ }^{[3,4,6]}$. Among those, innate and adaptive immune responses, vascular abnormalities, dermal microorganism imbalances, neurovascular dysregulation and environmental factors such as UV light have been considered to interact, resulting in chronic inflammation and the development of fibrosis $^{[3,4,7,8]}$. Furthermore, dysregulation of the skin microbiome resulting from different triggering factors has been considered 
to favor the colonization and multiplication of Demodex folliculorum which has been cited to cause inflammation in papules and pustules as well asgranulomas ${ }^{[9]}$. But, dysregulation of the microbiome and over-colonization by $D$. folliculorum may also influence the balanced presence of other microbes present on the skin. In this perspective, the role of Bacillus oleronius and Helibiobacterium pylori has been extensively reviewed by Yamasaki and Gallo in $2009^{[8]}$. In their review, the authors reported that B.oleronius was isolated from $D$. folliculorum and that the antigens reacting to sera from rosacea individuals but not from control individuals could be identified ${ }^{[10]}$. The extracts of the B.oleronius stimulated proliferation of mononuclear cells from patients with rosacea suggesting that rosacea individuals are exposed to the B.oleronius molecules and that B.oleronius from $D$. folliculorum induces inflammatory in rosacea. Conversely, the correlation of $H$. pylori infection and rosacea is controversial and inconsistent among clinical observation ${ }^{[11-14]}$. Several reports showed seropositivity to $H$. pylori in rosacea individuals. Eradication therapy for gastric $H$. pylori infection showed preferable outcome for rosacea symptoms though it is not clear if the improve of rosacea is due to $H$. pylori eradication ${ }^{[15-17]}$. The authors further raised the hypothesis that innate immunity and stimulation of toll-like receptors (TLR) may be caused by D. folliculorum leading to inflammation of the skin tissues ${ }^{[8,18]}$.

The development of rhinophyma, observed in stage III rosacea, remains poorly explained. The vascular abnormalities induce local production of transforming growth factor $\beta 1$ (TGF- $\beta 1$ ) capable of creating fibrosis and therefore cetaceous thickening ${ }^{[19]}$. Dissection of major players for disease progression is severely hindered by the complex activation of the innate and adaptive immune systems, enhanced neuroimmune communication, profound blood vessel and possibly lymphatic vessel changes, and activation of almost every resident cell in the $\operatorname{skin}^{[7,20]}$. Furthermore, elevated expression of vascular endothelial growth factor (VEGF) has been observed in the skin of patients with rosacea ${ }^{[21]}$. VEGF proliferates vascular endothelial cells as well as increase permeability of vessels.

Rosacea is not an isolated skin disease. It has been reported to be associated with allergies (airborne, food), respiratory diseases, gastroesophageal reflux disease, other gastrointestinal diseases, hypertension, metabolic and urogenital diseases, and female hormone imbalance. Compared with mild rosacea (stage I), moderate to severe rosacea (stage II to III) was significantly associated with hyperlipidemia, hypertension, metabolic diseases, cardiovascular diseases, and gastroesophageal reflux disease ${ }^{[22]}$.

Each subtype requires a different therapeutic ap$\operatorname{proach}^{[3-5]}$. Today, the management of rosacea is largely based on long-established treatments empirically tailored to the specific presenting symptoms and no real breakthrough has occurred to date $^{[23]}$. Topical treatments that are widely accepted are metronidazole and azelaic acid; agents under investigation that show promise include permethrin, calcineurin inhibitors and sulfur compounds. For systemic therapy, antibiotics (tetracyclines, macrolides) and recently doxycycline in anti-inflammatory rather than antimicrobial dosages are used, as well as isotretinoin in severe cases ${ }^{[24-26]}$. Furthermore, effective protection from UV light is recommended ${ }^{[26]}$.

The present study was conducted to assess the efficacy of different combinations of oral and topical medications in rosacea using histopathological, immunohistochemical and clinical methods. Regimens were adapted to patients with stage I or stage II/III rosacea; patients with ocular rosacea were not considered for this study. Basic combination regimens (BCR) included diosmine, a venotonic, sulpiride, a neuroleptic and pimecrolimus, and a calcineurin inhibitor to treat stage I rosacea; doxycycline, considered as an anti-inflammatory drug at beyond the recommended doses, sulpiride, adapalene, a retinoid, clindamycin, an oral antibiotic and pimecrolimus were used for patients with stage II/III rosacea. Both groups were compared after 16 weeks to patients receiving a complex combination regimen (CCR) consisting of the BCR treatment to which ademetionine, shown to regulate the vascular endothelial growth factor (VEGF) in cancer, was added ${ }^{[27,28]}$.

\section{Methods}

This randomized, open label, prospective and exploratory study was conducted from 2008 to 2013 at 3 investigational sites in Ukraine in male patients of at least 18 years of age. Prior to study initiation, approval from the local ethics committee and regulatory institutions was obtained and patients provided written informed consent. To be included in this study, patients had to suffer from a clinically confirmed erythematous stage I rosacea (mild erythema, small amounts of telangiectasia, absence of papules, pustules, nodes, plaques, rhinophyma), papulopustular stage II rosacea (persistent erythema, frequent telangiectasia, presence of papules, pustules) or stage III rosacea (persistent lasting erythema, frequent telangiectasia, papules, pustules, rarely - nodes, plaques, rhinophyma) according to Wilkin et $\mathrm{al}^{[34]}$.

Clinical evaluations included demographic and baseline disease characteristics as well as assessments of concomitant treatments to avoid drug interactions with the proposed treatment regimens. Clinical efficacy was based on clinical success, evaluated on a 4-step scale ranging from "complete recovery" to "no improvement" and on the quality of life assessed by the patients at baseline and after 16 weeks of treatment using an adapted version of the dermatological index of life quality (DIQL) proposed by Finlay et $\mathrm{al}^{[35]}$. Recurrence was assessed one year after the last treatment. Safety assessments included adverse events reporting and standard laboratory analyses.

To histologically compare the evolution of rosacea between baseline and the end of treatment period, skin biopsies were performed at inclusion and after 16 weeks of treatment in 12 patients with stage I rosacea and 11 patients with a clinically confirmed stage II rosacea; all patients provided written informed consent prior to this additional invasive procedure. Samples were obtained after local anesthesia with $0.5 \%$ solution of novocaine. Skin biopsies samples with a size of $0.3 \times 0.3 \times$ $0.3 \mathrm{~cm}$ were fixed in $10 \%$ neutral formalin solution and paraffin blocks. Samples were stained with hematoxylin and eosin. Pictures were made using a light microscope «Olympus AX-70Provis» (Olympus, Japan).

Immunohistochemical investigations (IHC) were made to assess the impact on the innate immunity. The following specific markers were assessed at baseline and week 16: CD4 +, $\mathrm{CD} 1 \mathrm{a}+, \mathrm{CD} 68+, \mathrm{CD} 34+, \alpha-\mathrm{SMA}, \mathrm{CD} 105+, \mathrm{CD} 138+$, MMP1 (Matrixmetallo proteinase), MMP-9, S100 and Vcl-2. VEGF markers were used to assess the activity of ademetionine on the vascular endothelial growth factor. Skin sample slices of 4.6 
mm thickness were fixed on special adhesive slides (SuperFrost Plus $^{\mathrm{TM}}$, Thermo Scientific, US). A semi quantitative method using a score going from $0=$ negative response (less than $5 \%$ staining cells) to $3=$ intense color (almost all cells stained positive) was used to assess the response. Proliferative activity was studied using the monoclonal antibody Ki-67.

Patients who met the inclusion criteria were randomized at the study sites in a 2 to 1 ratio to one of the 2 treatment regimens developed for their stage of the disease (Figure 1).
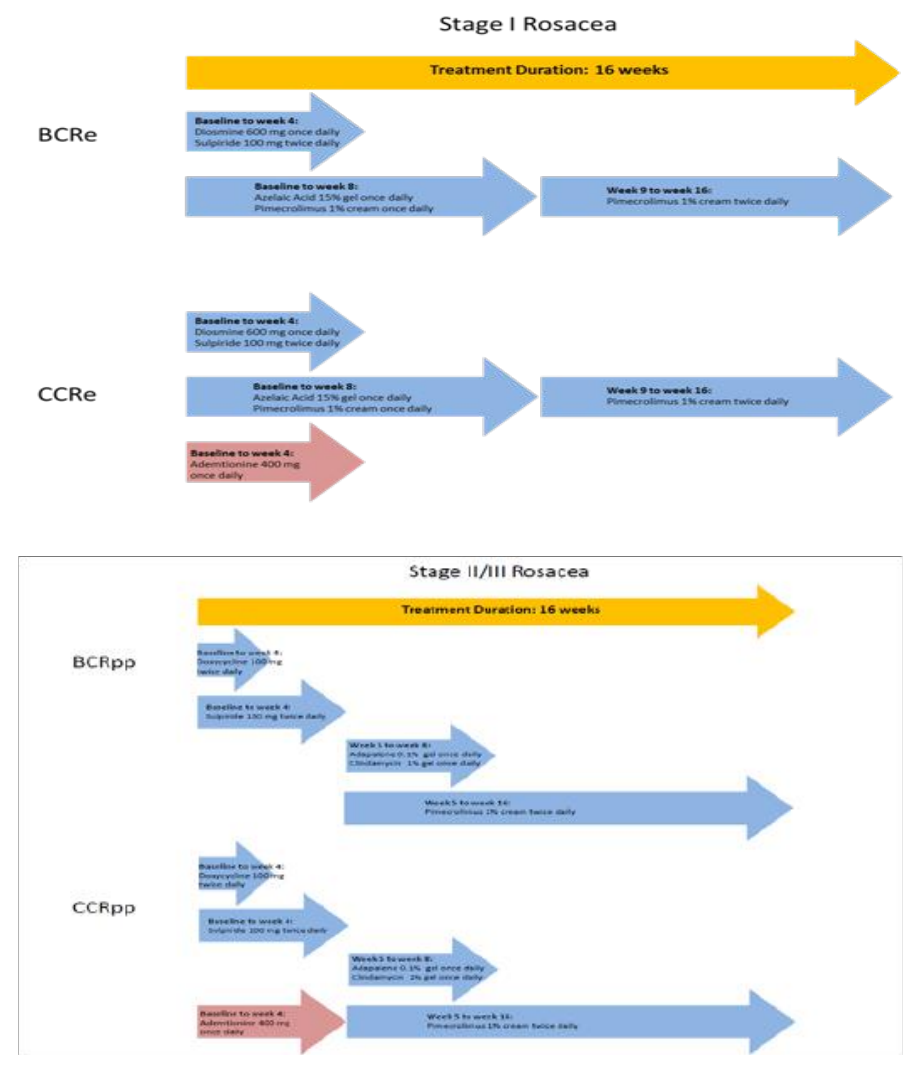

Figure 1: Treatment Regimens for Stage I and Stage II/III Rosacea.

For adjunctive skin care, all patients received micellar water during the treatment period, (Sensibio H2O, Bioderma, France), which was to be applied in the morning and evening using a cotton swab to clean the face and the skin around the eyes. An anti-erythema cream (Sensibio AR/AR BB cream, Bioderma, France) was to be applied once daily on the cleansed skin. Depending on the type of sebaceous excretions and, if necessary, patients were recommended to use a moisturizer (Sensibio Forte/Light, Bioderma, France) and a sunscreen (Photoderm AR, Bioderma, France) once daily. A total of 8 patients had a medical history of temporary exacerbation of seborrheic dermatitis in winter. From November to March, these patients received an anti-seborrheic cream (Sensibio DS cream, Bioderma, France) for their seborrheic areas. Furthermore, patients were allowed to use a soothing mask such as Sensibio Mask (Bioderma, France).

Statistics were performed using Microsoft Excel 2003 and Statistica v6.1 (StatsoftInc. USA). Basic statistical characteristics included: number of observations (n), means (M), standard error of average (m), 95\% confidence interval $(95 \%$ $\mathrm{CI}$ ), relative ratio (abs.\%). The parametric (Student t-test) and nonparametric criteria (Mann Whitney U-test, chi-square test of Pearson (Chi2)) were used for comparisons. The relationship between factors was calculated using Spearman's rank correlation coefficients (r). The critical level of statistical significance (p) was considered at $\leq 0.05$.

\section{Results}

A total of 110 patients with a mean age of $42.5 \pm 1.3$ years were included. Of those, 50 patients had stage I and 60 patients had stage II/III rosacea. The majority $(78 ; 70.9 \%)$ of the subjects was younger than 50 years. A total of 49 subjects $(44.6 \%)$ had suffered from rosacea for more than 5 years, 8 $(17.3 \%)$ of whom had suffered for more than 10 years.

All patients reported rosacea symptoms including redness and rash. In 102 patients these symptoms were permanent. Further demographic and disease characteristics are provided in Table 1.

Table 1: Demographic and Baseline Disease Characteristics

\begin{tabular}{|l|l|}
\hline Parameter & Study population \\
\hline Overall age (years) & $42.5 \pm 1.3(18-73)$ \\
\hline Mean (Min-Max) & $35.3 \pm 1.5$ \\
\hline Mean age: Erythematous & $43.2 \pm 1.9$ \\
\hline Mean age: Papular & $51.4 \pm 2.7$ \\
\hline Mean age: Pustular & $23(19.1)$ \\
\hline Phototype (n (\%)) & $87(79.1)$ \\
\hline I & $5.6 \pm 0.4(0.1-16)$ \\
\hline II/III & $3,1 \pm 0.4$ \\
\hline Disease duration (years) & $5.5 \pm 0.7$ \\
\hline Mean (Min-Max) & $9.1 \pm 1.0$ \\
\hline Mean duration: Erythematous & $50(45.5 \%)$ \\
\hline Mean duration: Papular & $48(43.6 \%)$ \\
\hline Mean duration: Pustular & $12(10.9 \%)$ \\
\hline Disease Stage (n (\%)) & $60(54.5 \%)$ \\
\hline Stage I & \\
\hline Stage II & \\
\hline Stage III & Stage II + Stage III \\
\hline
\end{tabular}

\section{Results from histopathology and IHC}

Histopathology at base line showed that for both types of rosacea, epithelial cells of the sweat and sebaceous glands, smooth muscle and endothelial cells of blood vessels, some fibroblasts of the dermis and mononuclear cells stained for the VEGF marker. The production of this receptor was higher in cells of inflammatory infiltrates of stage II/III rosacea (Figure 2). IHC showed significant differences $(p<0.05)$ between the intensity of IHC for CD4 +, CD1a +, CD68 +, $\alpha$-SMA, CD105 ,$+ \mathrm{Ki}-67+$ from skin samples of patients with different clinical forms of rosacea (Table 1). For CD34 +, MMP-1 + MMP-9 +, $\mathrm{S} 100$, a trend for an increased expression in patients with stage II/III rosacea compared to those with the stage I form was observed. Indicators for the activity of CD138 were comparable in both groups. In patients with stage II/III rosacea, the proliferative activity of epidermal cells $(\mathrm{Ki}-67)$ was twice as high as in patients with the stage I form $(\mathrm{p}<0.001)$. Complete results for IHC for all markers are provided in Table 2. For both clinical forms, the proliferative activity of epidermal cells in rosacea subjects significantly $(\mathrm{p}<0,001)$ differed from those of healthy 
subjects, which, according to scientific literature, equals $4.30 \pm$ 0.42 per 100 cells of the basal layer ${ }^{[29]}$.

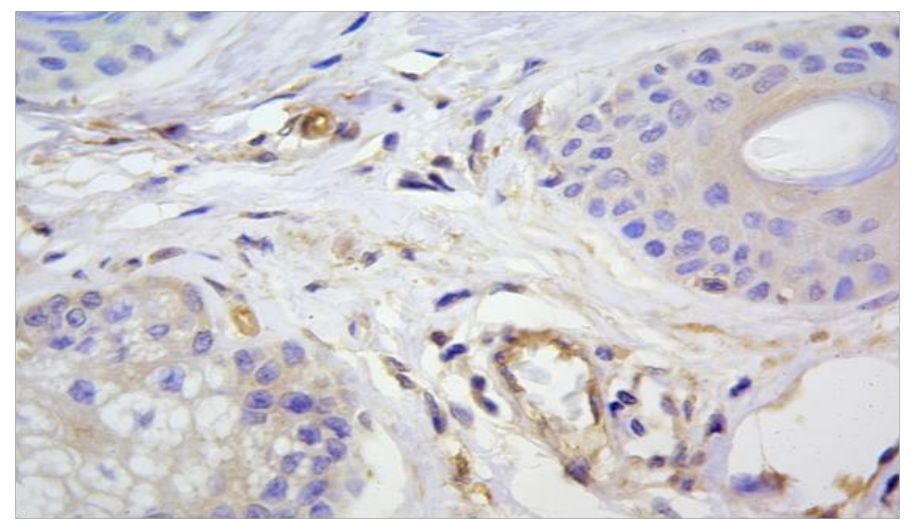

Figure 2: Positive cytoplasmic and membranous reaction with VEGF in epitheliocytes sebaceous glands, vascular endothelial and dermal single dendrotsytah. Immunohistochemical method, additional staining with Mayer hematoxylin Mayer.3b.x1000.

Table 2: Mean Values at Baseline forthe Expression of Immunity Markers.

\begin{tabular}{|l|l|l|c|}
\hline Marker & Stage I $(\mathbf{n}=\mathbf{1 2})$ & $\begin{array}{l}\text { Stage II/III } \\
(\mathbf{n}=\mathbf{1 1})\end{array}$ & $\begin{array}{l}\text { between groups } \\
\mathbf{p}-\mathbf{v a l u e *}\end{array}$ \\
\hline CD4 & $1.42 \pm 0.15$ & $1.91 \pm 0.09$ & $\mathbf{p}=\mathbf{0 . 0 1 5}$ \\
\hline CD1a & $0.75 \pm 0.13$ & $1.27 \pm 0.19$ & $\mathbf{p}=\mathbf{0 . 0 3 8}$ \\
\hline CD68 & $1.00 \pm 0.17$ & $1.64 \pm 0.15$ & $\mathbf{p}=\mathbf{0 . 0 1 6}$ \\
\hline CD34 & $2.00 \pm 0.25$ & $2.64 \pm 0.15$ & $\mathrm{p}=0.061$ \\
\hline $\boldsymbol{\alpha}-$ SMA & $1.25 \pm 0.13$ & $1.82 \pm 0.18$ & $\mathbf{p}=\mathbf{0 . 0 2 1}$ \\
\hline CD105 & $0.83 \pm 0.11$ & $1.55 \pm 0.28$ & $\mathbf{p}=\mathbf{0 . 0 2 6}$ \\
\hline CD138 & $0.58 \pm 0.15$ & $0.91 \pm 0.25$ & $\mathrm{p}=0.344$ \\
\hline MMP-1 & $0.25 \pm 0.13$ & $0.64 \pm 0.15$ & $\mathrm{p}=0.068$ \\
\hline MMP-9 & $2.58 \pm 0.15$ & $2.91 \pm 0.09$ & $\mathrm{p}=0.082$ \\
\hline VEGF & $1.83 \pm 0.21$ & $1.64 \pm 0.15$ & $\mathrm{p}=0.530$ \\
\hline S100 & $1.33 \pm 0.14$ & $1.73 \pm 0.14$ & $\mathrm{p}=0.065$ \\
\hline Ki-67 (y \%) & $11.41 \pm 0.21$ & $23.42 \pm 0.87$ & $\mathbf{p}<\mathbf{0 . 0 0 1}$ \\
\hline
\end{tabular}

* Mann-Whitney test

After 16 weeks of treatment in the stage I group, patients treated with CCRE showed a significant $(\mathrm{p} \leq 0.05)$ reduction of inflammatory infiltration, of the number of CD4 + cells and of T-helper cells. In the vessel and gland structure, the localization and number of cells that were positive for $\alpha$ SMA and CD34 had not significantly changed. Conversely, in the dermis, their number had decreased, indicating an inhibition of the myofibroblastic transformation and a positive treatment effect on the development of fibrosis. The most important changes were observed in the number of cells secreting MMP-1 and MMP-9 in the sub-epidermal layer of skin which were the most impacted by fibrotic changes. Reduction of the diameter of blood vessels of the dermis after treatment was partly due to a decrease of VEGF + cells, especially in the composition of infiltrates and dermal fibroblasts. A significant decrease $(p<0.05)$ in the proliferative activity of epidermal cells was observed. Estimated throughout was the decrease in the number of Ki-67 from $11.41 \% \pm 1.10$ at baseline to $7.02 \% \pm 1.91 \%$ at week 16 . There was no change in the number of Vcl-2 + cells, while the number of S-100 + cells had decreased in the dermis and papulopustular areas. In stage I patients who followed the BCRE regimen, the number of T-helper cells and infiltrations was reduced. The number of VEGF +, $\mathrm{Ki}-67+\mathrm{Vcl}-2+$ and S-100 + cells did not change significantly. In stage II/III patients, the CCRpp treatment achieved significantly $(\mathrm{p}<0.001)$ better results regarding immune markers, such as T-helper cells and macrophages, when compared with BCRpp. A significant $(p<0.001)$ reduction of the MMP activity and proliferative activity of epidermal cells from $23.42 \% \pm 1.87$ to $9.04 \% \pm 1.12$ was observed using the marker Ki-67, hypothesizing an improvement of the extracellular matrix.

\section{Clinical results}

After 16 weeks of treatment, the clinical success in both rosacea severity groups based on the investigator's assessment was significantly $(\mathrm{p} \leq 0.05)$ in favor of the CCR treatments compared to the basic combination regimens (Figure 3 ).

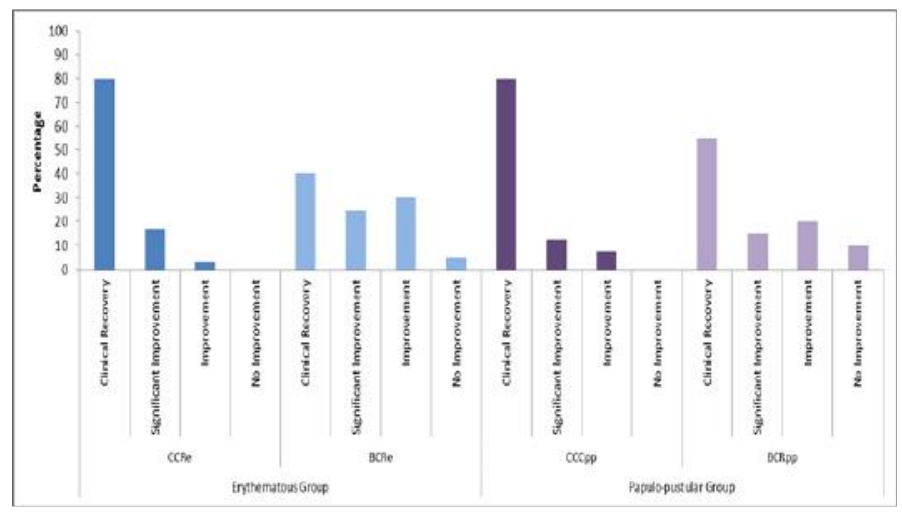

Figure 3: Clinical Success at Week 16

Both CCR treatments were statistically significantly superior $(\mathrm{p} \leq$ 0.05 ) to BCR treatments after 16 weeks of treatment based on the investigator's rating of clinical success.

At baseline, the total DIQL score was similar in all 4 treatment groups. Differences were statistically not significant. After 16 weeks of treatment, a statistically significant reduction $(p<0.001)$ of the total DIQL score was observed in both rosacea groups in favor of the CCR treatment (Figure 4).

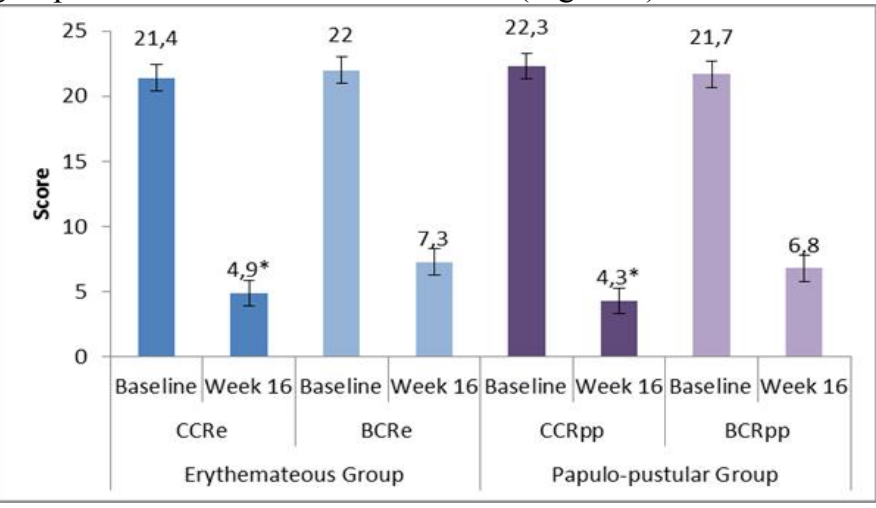

Figure 4: Daily Index of Quality of Life at Baseline and after 16 Weeks of Treatment.

$*: \mathrm{p} \leq 0.05$ in favor of CCR over BCR

Analysis of results one year after the last treatment showed that recurrence of stage I or stage II/III rosacea was significantly less frequent $(p<0.05)$ in patients who received the CCR treatment regimens (Table 3). 
Table 3: Results of Recurrence of Rosacea One Year after the Last Treatment.

\begin{tabular}{|l|l|l|l|l|}
\hline \multirow{2}{*}{} & \multicolumn{2}{|c|}{ Stage I Rosacea } & \multicolumn{2}{c|}{ Stage II/III Rosacea } \\
\cline { 2 - 5 } & $\begin{array}{l}\text { CCRe- } \\
\text { group } \\
\text { (n= 24) }\end{array}$ & $\begin{array}{l}\text { BCRe } \\
\text { group } \\
\text { (n= 17) }\end{array}$ & $\begin{array}{l}\text { CCRpp } \\
\text { group } \\
\text { (n= 31) }\end{array}$ & $\begin{array}{l}\text { BCRpp } \\
\text { group } \\
\text { (n= 15) }\end{array}$ \\
\hline Recurrence & $9(37.5 \%)$ & $13(76.5 \%)$ & $6(19.4 \%)$ & $8(53.3 \%)$ \\
\hline $\begin{array}{l}\text { Absence of } \\
\text { recurrence }\end{array}$ & $\mathbf{1 5 ( 6 2 . 5 \% )}$ & $4(23.5 \%)$ & $\mathbf{2 5 ( 8 0 . 6 \% )}$ & $7(46.7 \%)$ \\
\hline $\begin{array}{l}\text { between- } \\
\text { group } \\
\text { p-value }\end{array}$ & \multicolumn{3}{|c|}{$\mathbf{p}=\mathbf{0 . 0 1 4}$} & \multicolumn{2}{|c|}{$\mathbf{p = 0 1 9}$} \\
\hline
\end{tabular}

No systemic adverse events were observed during the study. Laboratory analysis results did not show any abnormal clinical values. Irritation was reported in 9 patients in the stage I patient group during the first 3 to 7 days of application of azelaic acid 15\% gel: 5 patients (16.7\%) in the CCRe group and 4 patients $(20 \%)$ in the BCRe group. No local adverse events were reported in the stage II/III patient group.

\section{Discussion}

Rosacea is a multifactorial and difficult to treat chronic dermatosis ${ }^{[1]}$. Its pathophysiology is not yet fully understood and a globally accepted treatment algorithm of the disease is still lacking. A large choice of topical and oral medications is available to treat rosacea comprising azelaic acid, ivermectine, brimonidine, metronidazole, tetracyclines and doxycycline to cite just a few ${ }^{[30-32]}$. However, none of them were shown to be able to manage the disease individually or to provide long-term relief and it seems as if combinations are the key to a more successful treatment of rosacea.

Over the past decades, several combinations including doxycycline and azelaic acid have been tested, especially in papulopustular rosacea, as reported by Bhatia and Del Rosso ${ }^{[33]}$. None were described for erythematous, stage I, rosacea. To fill this gap, the present clinical study assessed the clinical and histological impact of multiple combination treatments in association or not with ademetionine in the disease.

Results demonstrated that multiple combination regimens using topical and oral medications significantly improve stage I and stage II/III rosacea. Improvement on a histological, immunohistochemical and on a clinical level was superior when ademetionine was added to both regimens. Indeed, on an immunohistochemical level, the treatment effect on the innate immunity markers, which are expressed differently for the different rosacea stages, confirmed a reduction of CD4 + cells throughout, as well as showing, for the first time, changes in the number of $\mathrm{CD} 1 \mathrm{a}+, \mathrm{CD} 138+, \mathrm{CD} 68+$ and $\mathrm{Ki}-67+$ cells throughout. Furthermore, on a histological level, the treatment effect on the vascular component confirmed throughout a decrease in the density of blood vessels paralleled by the reduced expression of VEGF which could not be observed in the control groups. Conversely, no treatment effect was observed with BCR. Results from the clinical investigations as well as from the quality of life assessment after 16 weeks of treatment confirmed the superiority of a treatment with CCR. Results one year after the last treatment showed that relapse of rosacea had occurred less frequently with the combination including ademetionine than that without ademetionine.

All 4 investigated treatment regimens were well tolerated with few reports of transient irritation during the first 3 to 7 days after application of azelaic acid in patients with erythematous rosacea. This local side effect is well described for azelaic acid and did not have an impact on the patients' or investigator's decision to discontinue the study.

\section{Conclusion}

Results from the study confirmed that rosacea as a multifactorial disease needs a specific treatment for each stage. The question as to whether the chosen combination regimens act in a synergistic way or independently on each triggering factor of the disease cannot be answered in view of the present study results. We are aware that the unbalanced randomization in the different treatment groups, especially for patients with stage II/III rosacea, may be considered a limitation. However, overall treatment results do not raise this concern.

More detailed histological and immunohistochemical in vivo investigations will still be necessary to confirm our study findings. In addition, other research work will be necessary to confirm the role of ademetionine in the regulation of toll-like receptors stimulated by D.foliciculorum. Nevertheless, we showed that an integrated histological, immunohistochemical and clinical assessment of patients with rosacea prior to any treatment allows factors and changes to be detected which may affect the nature and severity of the disease.

By adding ademetionine to current rosacea treatments, the study showed new opportunities to treat rosacea by considering the clinical stage of the disease and allowing for a normalization of the level of vascular endothelial growth factor and of the proliferative activity of epidermal cells.

\section{Conflict of Interest: None to declare}

Financial Support: The authors did not receive any financial support for conducting this clinical investigation.

Acknowledgements: The authors acknowledge the editing support of Bioderma, France and the writing assistance of Karl Patrick Göritz, Scientific and Medical Writing Services, France.

\section{References}

1. Tidman, M.J. Improving the management of rosacea in primary care. (2014) Practitioner 258(1775): 27-30, 3.

2. Dirschka, T., Micali, G., Papadopoulos, L., et al. Perceptions on the Psychological Impact of Facial Erythema Associated with Rosacea: Results of International Survey. (2015) Dermatol Ther (Heidelb) 5(2): 117-127.

3. Preisz, K., Karpati, S. Rosacea: pathogenesis, clinical forms and therapy. (2010) Orv Hetil 151(30): 1209-1214.

4. Crawford, G.H., Pelle, M.T., James, W.D. Rosacea: I. Etiology, pathogenesis, and subtype classification. (2004) J Am Acad Dermatol 51(3): 327-341.

5. Weinkle, A.P., Doktor, V., Emer, J. Update on the management of rosacea. (2015) Clin Cosmet Investig Dermatol 8: 159-177.

6. Diamantis, S., Waldorf, H.A. Rosacea: clinical presentation and pathophysiology. (2006) J Drugs Dermatol 5(1): 8-12. 
7. Steinhoff, M., Schauber, J., Leyden, J.J. New insights into rosacea pathophysiology: a review of recent findings. (2013) J Am Acad Dermatol 69(6 Suppl 1): S15-S26.

8. Yamasaki, K., Gallo, R.L. The molecular pathology of rosacea. (2009) J Dermatol Sci 55(2): 77-81.

9. Abokwidir, M., Fleischer, A.B. Jr. Additional evidence that rosacea pathogenesis may involve demodex: new information from the topical efficacy of ivermectin and praziquantel. (2015) Dermatol Online J 21(9).

10. Lacey, N., Ni Raghallaigh, S., Powell, F.C. Demodex mites--commensals, parasites or mutualistic organisms? (2011) Dermatology 222(2): 128-130.

11. Rebora, A., Drago, F., Picciotto, A. Helicobacter pylori in patients with rosacea. (1994) Am J Gastroenterol 89(9): 1603-1604.

12. Diaz, C., O'Callaghan, C.J., Khan, A., et al. Rosacea: a cutaneous marker of Helicobacter pylori infection? Results of a pilot study. (2003) Acta Derm Venereol 83(4): 282-286.

13. Argenziano, G., Donnarumma, G., Iovene, M.R., et al. Incidence of anti-Helicobacter pylori and anti-CagA antibodies in rosacea patients. (2003) Int J Dermatol 42(8): 601-604.

14. Szlachcic, A. The link between Helicobacter pylori infection and rosacea. (2002) J Eur Acad Dermatol Venereol 16(4): 328-333.

15. Utas, S., Ozbakir, O., Turasan, A., et al. Helicobacter pylori eradication treatment reduces the severity of rosacea. (1999) J Am Acad Dermatol 40(3): 433-435.

16. Gedik, G.K., Karaduman, A., Sivri, B., et al. Has Helicobacter pylori eradication therapy any effect on severity of rosacea symptoms? (2005) J Eur Acad Dermatol Venereol 19(3): 398-399.

17. Boixeda de Miquel, D., Vazquez Romero, M., Vazquez Sequeiros, E., et al. Effect of Helicobacter pylori eradication therapy in rosacea patients. (2006) Rev Esp Enferm Dig 98(7): 501-509.

18. Yamasaki, K., Kanada, K., Macleod, D.T., et al. TLR2 expression is increased in rosacea and stimulates enhanced serine protease production by keratinocytes. (2011) J Invest Dermatol 131(3): 688-697.

19. Cribier, B. Pathophysiology of rosacea: redness, telangiectasia, and rosacea. (2011) Ann Dermatol Venereol 138(Suppl 3): S184-S191.

20. Dorschner, R.A., Williams, M.R., Gallo, R.L. Rosacea, the face of innate immunity. (2014) Br J Dermatol 171(6): 1282-1284.

21. Gomaa, A.H., Yaar, M., Eyada, M.M., et al. Lymphangiogenesis and angiogenesis in non-phymatous rosacea. (2007) J Cutan Pathol 34(10): 748-753.

22. Rainer, B.M., Fischer, A.H., Luz Felipe da Silva, D., et al. Rosacea is associated with chronic systemic diseases in a skin severity-depen- dent manner: Results of a case-control study. (2015) J Am Acad Dermatol 73(4): 604-608.

23. Gallo, R., Drago, F., Paolino, S., et al. Rosacea treatments: What's new and what's on the horizon? (2010) Am J Clin Dermatol 11(5): 299 303.

24. Sobottka, A., Lehmann, P. Rosacea 2009 : new advances in pathophysiology, clinical staging and therapeutic strategies. (2009) Hautarzt 60(12): 999-1009.

25. Scheinfeld, N., Berk, T. A review of the diagnosis and treatment of rosacea. (2010) Postgrad Med 122(1): 139-143.

26. Gross, H. Sunscreen, antibiotics, laser. What can you do against rosacea?. (2009) MMW Fortschr Med 151(51-52): 18-19.

27. Da, M.X., Zhang, Y.B., Yao, J.B., et al. DNA methylation regulates expression of VEGF-C, and S-adenosylmethionine is effective for VEGF-C methylation and for inhibiting cancer growth. Braz (2014) J Med Biol Res 30: 0:0.

28. Zhang, Y.B., Da, M.X., Yao, J.B., et al. S-Adenosylmethionine Inhibits Expression of Vascular Endothelial Growth Factor-C Protein and Cellular Proliferation in Gastric Cancer. (2015) Sichuan Da Xue Xue Bao Yi Xue Ban 46(3): 384-388.

29. Potekaeva, N.N., Araviyskaya, E.R., Sokolovsk, E.V. Acne and rosacea. (2007) Potekaeva NN, editor: Bean.

30. Deeks, E.D. Ivermectin: A Review in Rosacea. (2015) Am J Clin Dermatol 16(5): 447-452.

31. Holmes, A.D., Waite, K.A., Chen, M.C., et al. Dermatological Adverse Events Associated with Topical Brimonidine Gel 0.33\% in Subjects with Erythema of Rosacea: A Retrospective Review of Clinical Studies. (2015) J Clin Aesthet Dermatol 8(8): 29-35.

32. van Zuuren, E.J., Fedorowicz, Z., Carter, B., et al. Interventions for rosacea. (2015) Cochrane Database Syst Rev4: Cd003262.

33. Bhatia, N.D., Del Rosso, J.Q. Optimal management of papulopustular rosacea: rationale for combination therapy. (2012) J Drugs Dermatol 11(7): 838-844.

34. Wilkin, J., Dahl, M., Detmar, M., et al. Standard grading system for rosacea: report of the National Rosacea Society Expert Committee on the classification and staging of rosacea. (2004) J Am Acad Dermatol 50(6): 907-912.

35. Finlay, A.Y., Khan, G.K. Dermatology Life Quality Index (DLQI)-a simple practical measure for routine clinical use. (1994) Clin Exp Dermatol 19(3): 210-216.
Online ISSN: 2381-0858

Journal Title: Investigative Dermatology and Venereology Research Journal Short Name: Invest Dermatol Venereol Res
Ommega Online Publishers

E-mail: editor.dermatology@ommegaonline.org Website: www.ommegaonline.org 